\title{
Measurement of MMP Activity in Synovial Fluid in Cases of Osteoarthritis and Acute Inflammatory Conditions of the Knee Joints Using a Fluorogenic Peptide Probe-Immobilized Diagnostic Kit
}

\author{
Ju Hee Ryu1,2, \#, Aeju Lee 1, 3,\#, Myung Sook Huh¹, Junuk Chu¹, Kwangmeyung Kim¹, Byung-Soo Kim², \\ Kuiwon Choi ${ }^{1}$, Ick Chan Kwon ${ }^{1}$, Jong Woong Park ${ }^{4 \bowtie}$, Inchan Youn ${ }^{1 凶}$ \\ 1. Biomedical Research Center, Korea Institute of Science and Technology, Seongbuk-Gu, Seoul, South Korea; \\ 2. School of Chemical and Biological Engineering, Seoul National University, San 56-1, Sillim-dong, Gwanak-gu, Seoul 151-744, South \\ Korea; \\ 3. Department of Laboratory Medicine, College of Medicine, Korea University, Seoul, South Korea; \\ 4. Department of Orthopaedic Surgery, College of Medicine, Korea University, Seoul, South Korea. \\ \#These authors contributed equally to this paper.
}

Corresponding author: Dr. Inchan Youn, Biomedical Research Center, Korea Institute of Science and Technology, Seongbuk-Gu, Seoul, South Korea. Email: iyoun@kist.re.kr. Or Dr. Jong Woong Park, Department of Orthopaedic Surgery, College of Medicine, Korea University, Seoul, South Korea. Email: ospark@korea.ac.kr.

(c) Ivyspring International Publisher. This is an open-access article distributed under the terms of the Creative Commons License (http://creativecommons.org/ licenses/by-nc-nd/3.0/). Reproduction is permitted for personal, noncommercial use, provided that the article is in whole, unmodified, and properly cited.

Received: 2011.09.05; Accepted: 2011.10.22; Published: 2012.02.14

\begin{abstract}
Purpose: A fluorogenic peptide probe-immobilized diagnostic kit was used to analyze MMP activity in the synovial fluids (SFs) from patients with osteoarthritis (OA) and acute inflammatory conditions of the knee joint.

Methods: The MMP diagnostic kit containing a polymer-conjugated MMP probe immobilized on a 96-well plate was utilized for high-throughput screening of MMP activity in SFs from OA patients $(n=33)$ and patients with acute inflammatory conditions of the knee joint $(n=5)$.

Results: Compared to SF from OA patients, SF from patients with acute inflammatory conditions of the knee joint presented stronger NIR fluorescent signals. In gelatin zymography, most samples from patients with acute inflammatory conditions of the knee joint also displayed $92 \mathrm{kDa}$ (pro-form) MMP-9 and faint $84 \mathrm{kDa}$ (active form) MMP-9, while SF from OA patients did not display detectable MMP-9 activity .

Conclusion: The presence of a strong fluorescence signal from the MMP diagnostic kit corresponded well with patients with acute inflammatory conditions of the knee joint. The results suggest that our MMP diagnostic kit can be useful in differentiation between early stages of OA and acute inflammatory conditions of the knee joint.
\end{abstract}

Key words: Matrix metalloproteinases, Osteoarthritis, Synovial fluid, Diagnostic kit, Acute inflammatory conditions of the knee joint

\section{Introduction}

Osteoarthritis $(\mathrm{OA})$ is the most common degenerative joint disease, and affects about $12 \%$ of the population [1]. OA is a chronic, disabling disease in- volving progressive cartilage destruction accompanied by intractable pain, and most frequently occurs in the knee joint. Abnormal mechanical injury and 
stress from repeated impact loading on the joint are involved in the progression of OA. Digestive proteases are detected in larger amounts in OA cartilage than in normal cartilage $[2,3]$. Current diagnosis of OA mainly focuses on two major points. The clinician must first differentiate OA from other types of arthritis. It is also important to determine whether a patient has primary OA or a secondary form of OA that is associated with other diseases.

OA progression is currently diagnosed by physical examination, various imaging studies, and laboratory tests. Any symptoms associated with OA, such as joint effusion, tenderness, crepitation, decreased range of motion, and alignment of the knee joints, are carefully evaluated by physician. Radiographs typically show joint space narrowing, subchondral sclerosis, marginal osteophytes, and, sometimes, subchondral cyst formation [4]. In diagnosis of OA, laboratory tests (e.g. blood analysis and synovial fluid analysis) are not routinely requested by physicians because there are no specific biologic markers for OA. When OA patients complain about joint effusions combined with unusual pain, differential diagnosis is mandatory in order to rule out acute inflammatory conditions, such as gout, rheumatoid arthritis, pyogenic arthritis, or joint effusions secondary to trauma that violate intra-articular structures of the joints. There are several differential diagnostic methods for use in ruling out acute inflammatory conditions with $\mathrm{OA}$; however, many of these laboratory tests and imaging studies are significantly time-consuming. Due to its insignificant change on radiographs, accurate diagnosis of joint effusion combined with early stages of $\mathrm{OA}$ using conventional methods in the out-patient clinic is difficult $[5,6]$. In addition, diagnosis of early stage OA, especially accompanying joint effusion, can be confused with joint effusion caused by traumatic knee joint injury, such as anterior cruciate ligament rupture and/or meniscus tear. Because $\mathrm{X}$-rays provide no information, additional studies are recommended, including joint fluid aspirate analyses or MRIs, which are time-consuming and additional economic burdens to patients. Ready availability of a specific biological marker for $\mathrm{OA}$ at the bedside would allow more individual patient-customized therapy $[7,8]$.

Among various biological markers associated with OA, matrix metalloproteinases (MMPs) play a primary role in cartilage degradation in human joint disease and function downstream of $\mathrm{OA}$ signaling pathways $[9,10]$. Following excretion from the cell as inactive pro-forms, MMPs are activated into the active enzyme, which can be inhibited by reversible binding with specific inhibitors, tissue inhibitors of metallo- proteinases (TIMPs) [11]. Activation of MMPs is closely related to cartilage degradation. One or more MMPs can digest most of the matrix components in vitro [12]. Elevated levels of MMPs are found in OA cartilage at the site of cartilage destruction and specific digested parts of MMPs are present in SF samples from OA patients [13]. MMP-2 and -9 are especially important in cartilage degradation because they can degrade a variety of collagens, including basement membrane, type $\mathrm{V}$ collagen, and denatured fibrillar type I collagen $[14,15]$. MMPs could potentially be used as a promising biological marker of OA.

Several methods have been used for detection of MMP activity in OA SF. These methods include enzyme-linked immunosorbent assay (ELISA), gelatin zymography, and Western blotting. ELISA has been frequently used for measurement of total amounts of pro-form MMPs plus MMP-TIMP complexes, whereas measurement of MMP in the active form is more appropriate in assessment of the potential of matrix degradation from MMPs [11]. In terms of sensitivity, gelatin zymography and Western blotting are superior in their detection of MMPs; however, due to time-consuming and cumbersome practices, they are unsuitable for rapid high-throughput screening of larger samples.

Previously, we developed a new MMP diagnostic kit for high-throughput screening of MMP bioactivity using polymeric MMP probes [16]. Polymeric MMP probes chemically conjugated onto the plate surface exhibited their excellent fluorogenicity against target MMPs. In this study, we analyzed MMP activity in SF samples from patients with OA and acute inflammatory conditions of the knee joints using our MMP diagnostic kit, and compared the results with those of gelatin zymography and Western blotting.

\section{Materials and methods}

\section{Harvesting Synovial Fluids}

OA of the knee joint was diagnosed by physical and radiological evaluations based on the American College of Rheumatology criteria [17]. OA patients were subdivided into four groups according to the severity of disease progression based on the Kellgren-Lawrence Grading Scale: Grade 1: doubtful narrowing of joint space and possible osteophytic lipping, Grade 2: definite osteophytes, definite narrowing of joint space, Grade 3: moderate multiple osteophytes, definite narrowing of joint space, some sclerosis and possible deformity of bone contour, Grade 4: large osteophytes, marked narrowing of joint space, severe sclerosis and definite deformity of bone contour [18]. Grades of OA were separately scored 
and statistically confirmed by three physicians using a cross-validation method. All of the patients enrolled in the study complained of joint pain and noticeable joint effusions. Prior to aspiration of the joint fluids, informed consents were obtained from each patient according to the approved recommendation of the institutional review board (IRB) of the Korea University College of Medicine. SFs were aspirated from the knee joint of these patients in order to relieve their symptoms and the aspirates were aliquoted in $2 \mathrm{ml}$ samples and stored at $-70^{\circ} \mathrm{C}$ prior to use. SFs were diluted with phosphate buffered saline (PBS) of pre-optimized quantity for respective experiments and centrifuged at $10,000 \mathrm{~g}$ for $10 \mathrm{~min}$ at $4{ }^{\circ} \mathrm{C}$ for removal of any flocculate components.

\section{Preparation of the matrix metalloproteinase diagnostic kit}

The MMP diagnostic kit was prepared by immobilization of a polymer-conjugated MMP peptide probe onto each well of a maleic anhydride-activated 96-well plate (Pierce), as previously described [16]. In summary, for construction of the MMP diagnostic kit, first, MMP peptide probes consisting of near infrared (NIR) fluorochrome, Cy5.5 (ex/em; 675/695 nm), MMP-2,9,13 substrate peptides (Gly-Pro-Leu-Gly-Val$\operatorname{Arg}($ pbf)-Gly-Lys(boc)-Gly-Gly, the cleavage site between Gly and Val), and black hole quencher-3 (BHQ-3, abs. $650 \mathrm{~nm}$ ) were chemically conjugated to glycol chitosan (GC) polymers, resulting in polymer-conjugated MMP peptide probes [19]. Second, the polymer-conjugated MMP peptide probes were effectively immobilized onto each well of a maleic anhydride-activated 96-well plate, resulting in an MMP diagnostic kit.

\section{Enzyme specificity of the polymer-conjugated MMP peptide probe}

Specificity of the polymer-conjugated MMP peptide probe was examined by incubation with activated MMP-2, MMP-3, MMP-7, MMP-9, and MMP-13, as previously described [11]. In summary, the polymer-conjugated MMP peptide probe $(20 \mathrm{nM})$ was examined by incubation in the reaction buffer ( 50 mM TRIZMA $\cdot \mathrm{HCl}, 10 \mathrm{mM} \mathrm{CaCl} 2 \cdot 2 \mathrm{H}_{2} \mathrm{O}, 0.15 \mathrm{M} \mathrm{NaCl}$, and $0.05 \%$ Brij35, pH 7.8) containing $15 \mathrm{nM}$ of activated MMP-2, MMP-3, MMP-7, MMP-9, and MMP-13 (R\&D Systems, Inc., Minneapolis, MN), respectively. MMP-2, MMP-3, MMP-7, and MMP-13 enzymes were activated by incubation with $2.5 \mathrm{mM}$ of p-aminophenyl mercuric acid in $0.1 \% \mathrm{NaOH}$ buffer for $1 \mathrm{~h}$ at $37^{\circ} \mathrm{C}$ prior to initiation of the assay. Fluorescence intensity was measured using a spectrofluorometer (F-7000 Fluorescence Spectrophotometer,
Hitach, Tokyo, Japan) $80 \mathrm{~min}$ after incubation at $37^{\circ} \mathrm{C}$. The excitation wavelength was set at $675 \mathrm{~nm}$ and emission spectra were recorded from 676 to $800 \mathrm{~nm}$ [20].

\section{Screening of MMP activity in SF from OA pa- tients}

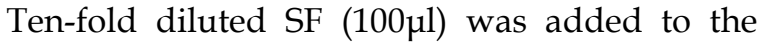
MMP diagnostic kit and incubated for $1 \mathrm{~h}$ at $37^{\circ} \mathrm{C}$ for measurement of MMP activity. PBS buffer was regarded as control. Following incubation, the MMP diagnostic kit was imaged using a 12-bit CCD camera (Kodak, Japan) equipped with a special C-mount lens and a Cy5.5 bandpass emission filter (680 to $720 \mathrm{~nm}$; Omega Optical, Brattleboro, VT). Fluorescence intensity was measured using a fluorescence spectrofluorometer.

\section{Zymography and image analysis}

Five-fold diluted SF $(5 \mu l)$ was mixed with an equal volume of sample buffer ( $10 \%$ sodium dodecyl sulfate, $0.5 \mathrm{M}$ Tris $[\mathrm{pH} 6.8]$, and $5 \%$ [v/v] glycerol containing $0.1 \%[\mathrm{w} / \mathrm{v}]$ bromphenol blue). For gelain zymography, the SF sample was loaded on a $10 \%$ polyacrylamide gel containing copolymerized gelatin $(1 \mathrm{mg} / \mathrm{ml})$. The gel was washed two times in $2.5 \%$ (v/v) Triton X-100 (30min and $1 \mathrm{~h}$ each) and incubated in $50 \mathrm{mM}$ Tris $\mathrm{HCl}, \mathrm{pH} 7.5,0.2 \mathrm{M} \mathrm{NaCl}$, and $10 \mathrm{mM}$ $\mathrm{CaCl} 2$ at $37^{\circ} \mathrm{C}$ for $24 \mathrm{~h}$. The gel was stained with $3 \%$ Coomassie blue and destained. MMP activity was revealed as clear bands on a dark background of uniform light blue staining for comparison of gelatinase molecular weight standards [21]. After development, gel images were captured using DNR MiniBIS pro (Bio-imaging systems).

\section{Protein electrophoresis and Western blotting}

SF $(30 \mu l)$ mixed with sample buffer $(0.8 \%$ sodium dodecyl sulfate, $0.25 \mathrm{M}$ Tris [pH 6.8], and $10 \%$ [v/v] glycerol containing $0.05 \%[\mathrm{w} / \mathrm{v}]$ bromphenol blue) (final dilution of SF: 15 -fold) ran on $7.5 \%$ polyacrylamide gel after $10 \mathrm{~min}$ of boiling. Gels were then transferred to a blotting membrane using iBLOT (Invitrogen). Membranes were blocked with 5\% low fat dry milk in Tris bufferd saline-Tween20 (TBST) for $1 \mathrm{~h}$ at room temperature (RT), followed by incubation with primary antibody (anti-MMP-13 [Calbiochem, 1:400] at RT for $1 \mathrm{~h}$. Thereafter, 3 washes (15 min each) with TBST were performed and membranes were incubated with a 1/2000 dilution of horseradish peroxidase-conjugated anti-mouse antibody (Waters) in TBST for $30 \mathrm{~min}$, followed by further washings (3 washes, $15 \mathrm{~min}$. each) with TBST. Immunoreactive proteins were visualized using enhanced chemiluminescence (Pierce). 


\section{Statistical Analyses}

Data were obtained from triplicate samples and expressed as mean \pm standard deviations. One-way analysis of variance (ANOVA) was used for comparison of continuous variables between groups. A value of $p<0.05$ was considered statistically significant.

\section{Results}

The MMP diagnostic kit was fabricated for high-throughput screening of MMP-2, -9 , and -13 bi- oactivity in SF samples of OA patients in a rapid and easy fashion (Fig. 1A). The principle of the MMP diagnostic kit is based upon recovery of quenched fluorescence by cleavage of specific substrate peptides. Once target enzymes, such as MMPs, cleave specific substrate peptides, the fluorophore becomes accessible for detection. Fluorescence intensity of the resulting product correlating with MMP activity is measured [16].

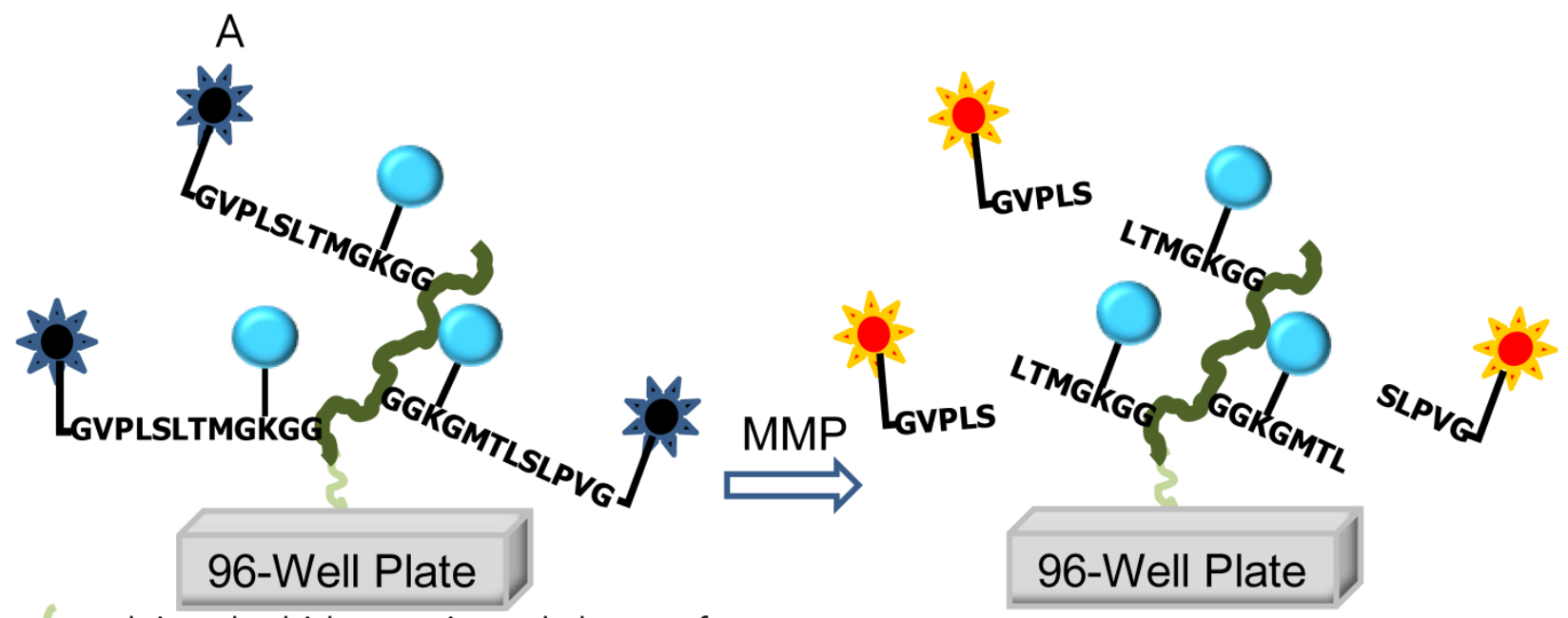

maleic anhydride-terminated plate surface

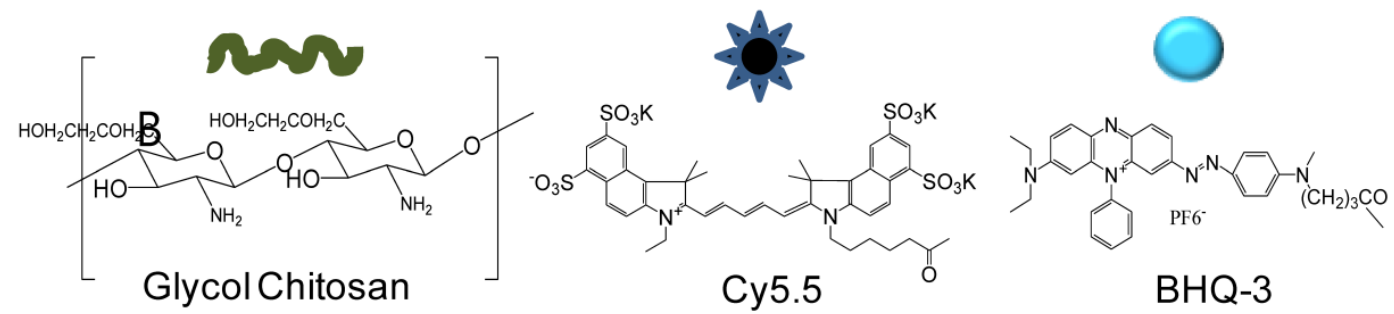

B

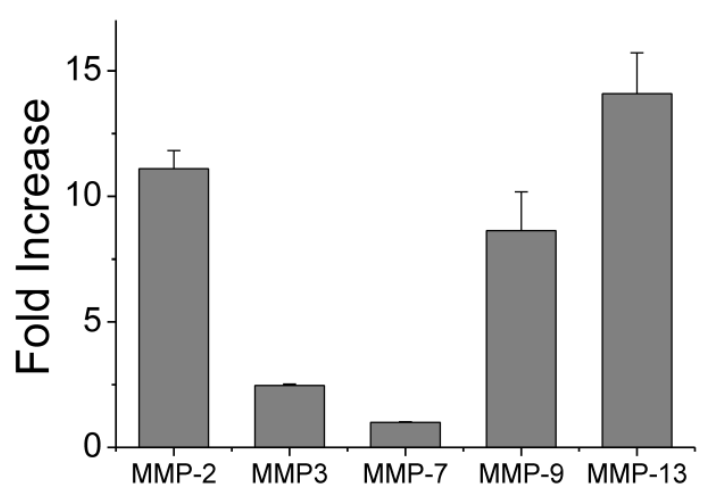

Figure I. (A) Schematic diagram of the MMP diagnostic kit for rapid and easy detection of MMP activity. When MMP-2, 9, I3 were simply added and incubated in the MMP diagnostic kit, the fluorescence of each well was recovered and the fluorescence intensity showed distinct differences within minutes through the NIR fluorescence imaging system. (B) Fluorescence intensity of polymer-conjugated MMP peptide probe in solution $(20 \mathrm{nM})$ in the presence of various stimuli (MMP-2, -3, -7, -9, - I3) for 80 min at $37^{\circ} \mathrm{C}$. 
Table I. Characteristics of the patients and their knees. Results are shown as mean (SD), (median), and range

\begin{tabular}{llllll}
\hline Sex $\left(\mathrm{F} / \mathrm{M}^{*}\right)$ & Age (years) & Disease Duration (years) & Aspirated SF* volume $(\mathrm{ml})$ & Disease (Acute* $/ \mathrm{OA})$ & $\begin{array}{l}\text { Grading Scale } \\
(1 / 2 / 3 / 4)\end{array}$ \\
\hline $33 / 5$ & $57.6(15.3)$ & $4.63(6.85)$ & $5.8(6.4)$ & $5 / 33$ & $9 / 12 / 6 / 11$ \\
& $(60.5)$ & $(6.5)$ & $(3.0)$ & \\
& $19-84$ & $0-30$ & $0.1-30$ & \\
\hline
\end{tabular}

*F=female; M-male; $\mathrm{SF}=$ synovial fluid; Acute=acute inflammatory conditions of the knee joints.

\section{Acute $\quad O A, 1$ grade $O A, 2$ grade $O A, 3$ grade $O A, 4$ grade}
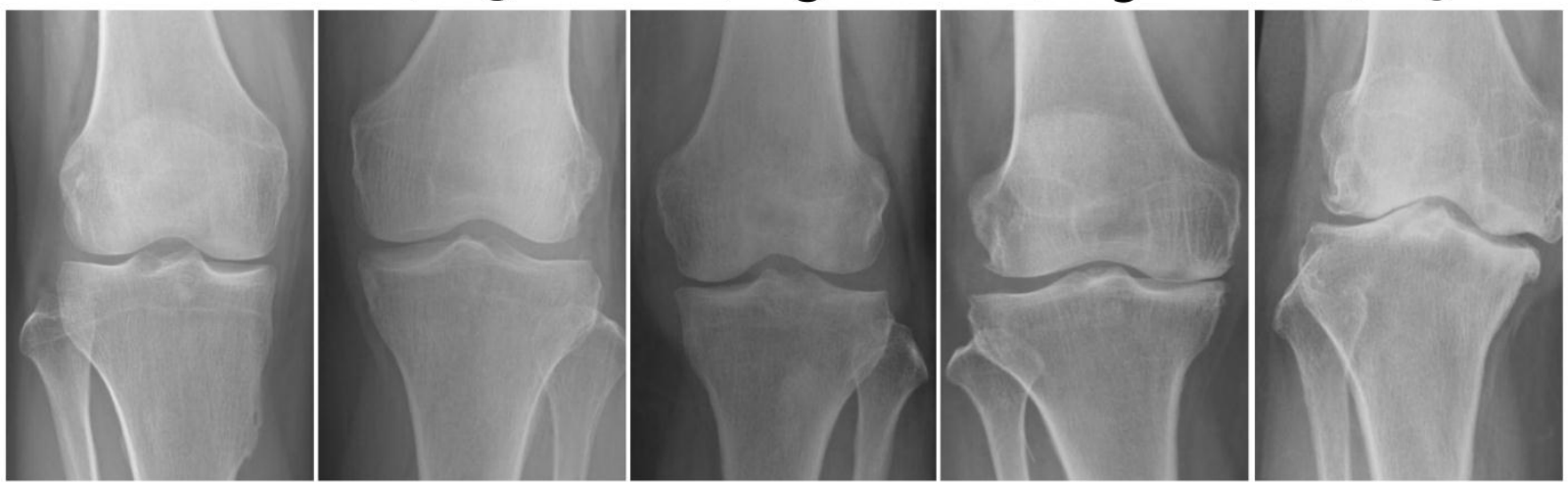

Figure 2. X-ray data from patients with OA (grades I -4) and acute inflammatory conditions of the knee joints.

For preparation of a high-throughput MMP diagnostic kit, first, the MMP peptide probe, which consisted of NIR fluorophore, MMP substrate peptide, and black hole quencher, was chemically conjugated to glycol chitosan (GC) polymer, in the resulting polymer-conjugated MMP peptide probe. Second, polymer-conjugated MMP peptide probes were effectively immobilized onto each well of maleic anhydride-activated 96-well plates, in the resulting MMP diagnostic kit. The polymer-conjugated MMP probe clearly demonstrated that significant recovery of fluorescence signals occurred against MMP-2, -9 , and -13 (11.1 $\pm 0.72,8.6 \pm 1.5$, and 14.1 \pm 1.6 -folds for MMP-2, -9 , and -13 , respectively) (Fig. 1B). A small amount of recovered fluorescent intensity occurred in the polymer-conjugated MMP probe treated by MMP-3 and MMP-7 80 min after incubation at $37^{\circ} \mathrm{C}$.

Among a total of 38 OA patients enrolled in this study, there were 33 females and 5 males (Table 1). The mean age was approximately 57.6 years and the mean disease duration was 4.63 years. Knee OA was diagnosed by clinical and radiological evaluations and divided into four groups according to the severity of OA based on the Kellgren-Lawrence radiographic grading scale, which emphasizes joint space narrowing and significance of osteophytes (grades 1 - 4, with 1 being early stage of OA and 4 severe OA). As OA progresses, the medial compartment becomes narrower and the lateral compartment becomes wider. There are also a number of osteophytes and large subchrondral cysts where the bones rubbed together. Early stages of OA are easy to distinguish from late stages of OA through X-rays (Fig. 2). However, differentiation between early stage $\mathrm{OA}$ and acute inflammatory conditions of the knee joint only through radiological evaluation is difficult. In fact, based on the radiologic findings, all 38 patients were initially diagnosed as OA combined with variable amounts of joint effusion. However, through further laboratory and diagnostic imaging studies, including MRI, 5 patients were finally diagnosed with acute inflammatory conditions of the knee joint, which can be combined with joint effusion.

The MMP diagnostic kit was used for detection of MMP activity in SF from all 38 patients (Fig. 3A). When the 10-fold diluted SF (100 $\mu \mathrm{l})$ was simply incubated in the MMP diagnostic kit at $37^{\circ} \mathrm{C}$, each well showed distinct NIR fluorescent signal differences after 1 hour post-incubation. Recovered NIR fluorescent signals were not dependent on OA severity, indicating that MMP activity in SF does not correspond to OA grade. Importantly, SF from patients with acute inflammatory conditions of the knee joint presented stronger NIR fluorescent signals, compared to OA 
patients, wherein a 3.8-fold increase in NIR fluorescent signals was observed (Fig. 3B). Median levels of recovery fluorescence signals were as follows: OA 127 (range from 21 to 238) and acute inflammatory condi- tions of the knee joints 483 (range from 247 to 679). Recovery fluorescence signals in the group with acute inflammatory conditions of the knee joint were significantly higher $(\mathrm{p}<0.05)$ than in the OA group.
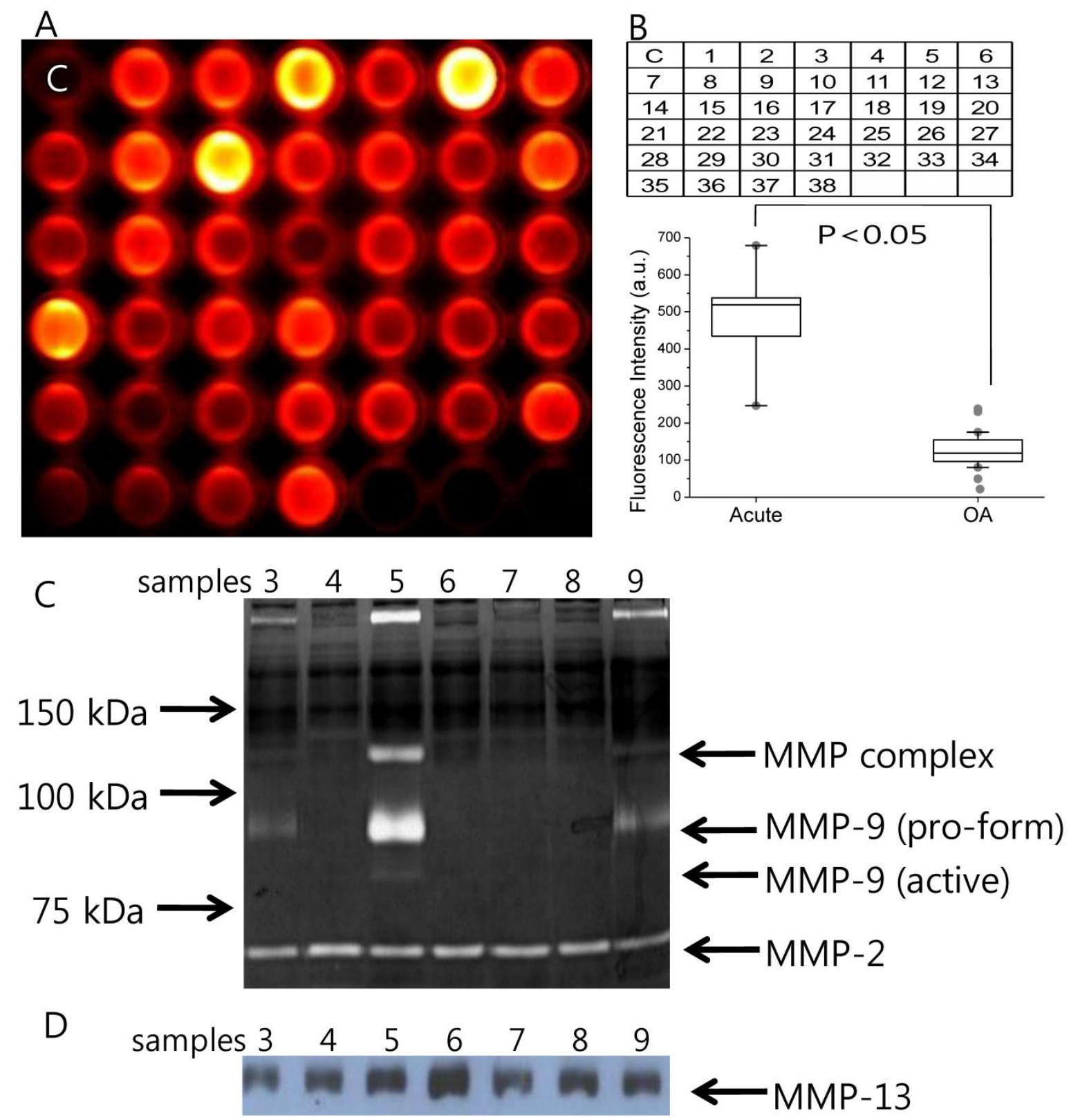

Figure 3. (A) NIR fluorescent image of the MMP diagnostic kit treated with SF samples from patients with OA and acute inflammatory conditions of the knee joints. Sample number 3, 5, 9, 13, and 2I are SF samples from patients with acute inflammatory conditions of the knee joints and $C$ indicates the well treated with PBS as control. (B) upper: Number of SF samples treated in (A). lower: Fluorescent intensity of the MMP diagnostic kit treated with SF samples from patients with OA and acute inflammatory conditions of the knee joints. Boxes show the median as well as the $25^{\text {th }}$ and $75^{\text {th }}$ percentiles, while whiskers indicate the $10^{\text {th }}$ and $90^{\text {th }}$ percentiles. Each circle represents an outlier. SF samples from 4 OA patients (lanes 2, 4-6) and 3 patients with acute inflammatory conditions of the knee joints (lanes I, 3, 7) analyzed by (C) gelatin zymography and (D) Western blotting using the MMP-I3 antibody. The positions of MMP-2 and MMP-9 are shown. Precision Plus Protein Standards (Bio-rad) were used as molecular size markers (data not shown). Sample number 3, 5, and 9 are SF samples from patients with acute inflammatory conditions of the knee joints. 
To measure amounts of individual MMP in SF from patients, gelatin zymography and Western blotting using the MMP-13 antibody were performed. Results of gelatin zymography revealed MMP activity at $72 \mathrm{kDa}$ and $92 \mathrm{kDa}$ (Fig. 3C). MMP activity at 72 $\mathrm{kDa}$ and $92 \mathrm{kDa}$ has been known to represent MMP-2 and MMP-9, respectively [22]. The presence of MMP-2 in all samples without clear differences in band intensities may represent constitutive expression of MMP-2. Most samples from patients with acute inflammatory conditions of the knee joint also displayed $92 \mathrm{kDa}$ (pro-form) MMP-9 and faint $84 \mathrm{kDa}$ (active form) MMP-9, while SF from OA patients did not display detectable MMP-9 activity. SF samples were subjected to Western blotting using the MMP-13 antibody. Results of Western blotting indicated that MMP-13 was detectable in all of the SF from patients with $\mathrm{OA}$ and acute inflammatory conditions of the knee joint. The presence of a strong fluorescence signal from the MMP diagnostic kit corresponded well with patients with acute inflammatory conditions of the knee joint. The results suggest that our MMP diagnostic kit can be useful in differentiation between early stages of OA and acute inflammatory conditions of the knee joint.

\section{Discussion}

Detection of MMP activity in biological samples provides important information for diagnosis, prognosis, and therapeutic monitoring of various diseases. For high throughput screening, rapid and continuous measurement is advantageous. In the current study, we have used the MMP diagnostic kit containing a polymer-conjugated MMP probe immobilized on a 96-well plate for detection of MMP activity in SF samples from patients with OA and acute inflammatory conditions of the knee joint. Compared to SF from OA patients, SF from patients with acute inflammatory conditions of the knee joints presented stronger NIR fluorescent signals. Measurement of active MMP using our MMP diagnostic kit could be useful in discriminating between early stages of OA and acute inflammatory conditions of the knee joint in a rapid and easy fashion. Also, this technology could be clinically applicable in diagnosing whether joint effusion is caused by primary OA or by inflammation secondary to other disease entities.

SF from patients with acute inflammatory conditions of the knee joint emitted noticeable NIR fluorescent signals, compared to those of primary OA patients. Comparing NIR fluorescent signals in the MMP diagnostic kit and zymography/Western blotting, expression of MMP-2 and -13 might be responsible for background signals in the SF sample; how- ever, MMP-9 appears to be one of the main MMPs expressed in cases of acute inflammatory conditions of the knee joints. We found statistically enhanced fluorescent signals in SF samples from the group with acute inflammatory conditions of the knee joint, compared to the OA group. We also found that fluorescent signals in SF samples from patients whose disease duration was less than 2 years $(222 \pm 158)$ were stronger than those from patients whose disease duration exceeded 2 years $(94.0 \pm 35.2)$ (Fig. 4$)$.

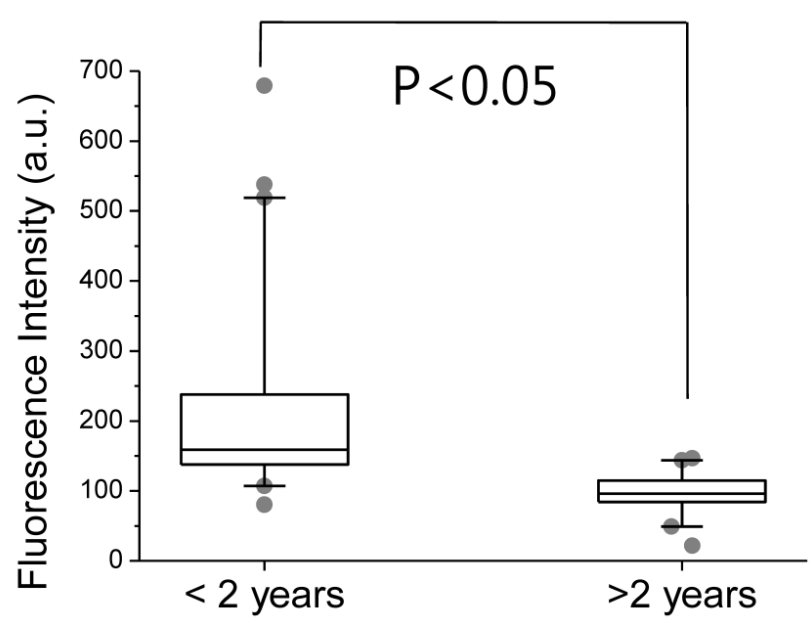

Figure 4. Fluorescent intensity of the MMP diagnostic kit treated with SF samples from patients whose disease duration is greater than and less than 2 years. Boxes show the median as well as the $25^{\text {th }}$ and $75^{\text {th }}$ percentiles, while whiskers indicate the $10^{\text {th }}$ and $90^{\text {th }}$ percentiles. Each circle represents an outlier.

$\mathrm{SF}$ is closer to the location of joint cartilage and protease degradation than serum or urine, and, therefore, could emit better local pathologic processes in the joint [23]. When SF aspiration was attempted from the knee joints of patients, it was found that aspirated SF samples varied in their viscosity and volume. In our experimental setup, small amounts of harvested SF $(10 \mu \mathrm{l})$ were applied to measure the concentration of MMP from the harvested SF sample; however, this method had limitations in presenting the total protein amount involved in biological activities in the joint. Therefore, estimation of SF volume for determination of total amounts of MMP in the joint would be desirable. Several studies have reported on possible methods for estimation of SF volume with radioisotopic dilution techniques that include [113In]-indium chloride or sodium [ $\left.{ }^{99} \mathrm{Tc}\right]$-pertechnetate and a non-radioactive method based upon albumin dilution upon intra-articular injection of saline [24]. Considering estimated SF volume to NIR fluorescent 
signals in the MMP diagnostic kit, the concentration of active MMP in the SF might be calculated with greater accuracy.

In this study, MMP acts as a biological marker for OA. Biological OA markers may allow detection of early and subtle changes in tissue at the molecular level. In addition to MMP, there are other proteases that may also be involved in the pathogenesis of OA, and can serve as biomarkers. For example, cathepsin and aggrecanase are known to play key roles in cartilage degradation. The diagnostic kit can be extended to other proteases, such as aggrecanase, simply by changing the specific peptide substrate linker between the fluorophore and black hole quencher. In addition, because MMPs are a homologous family of enzymes that possess high sequence similarity and similar three-dimensional structures, our MMP probe used in this diagnostic kit is specific to MMP-2, -9 , and -13 [25]. These properties may hamper MMP probes that are designed for high sensitivity and specificity to certain diseases over-expressing certain MMPs. To overcome this drawback, the use of multi-probes, including more than one MMP substrate sequence, may enhance sensitivity and specificity for certain types of MMPs.

In summary, we detected MMP activity in a large number of SF samples from patients with OA and acute inflammatory conditions of the knee joint using the MMP diagnostic kit containing a polymer-conjugated MMP probe immobilized on a 96-well plate in a rapid and easy fashion. The MMP diagnostic kit may be useful in discriminating between early stages of $\mathrm{OA}$ and acute inflammatory conditions of the knee joint, which can be difficult to tell apart if the diagnosis is made only based on morphological changes of the knee joints that appear on X-rays. Our diagnostic kit was also applied for detection of MMP activity in various biological samples, such as blood serum from rheumatoid arthritis patients. In addition, it can be utilized to distinguish pre-clinical diseases related to MMPs and in assessment of individual therapeutic monitoring of drug treatments and surgical therapy in a rapid and easy fashion.

\section{Acknowledgments}

This work was financially supported by the Real-Time Molecular Imaging Project and GRL Program of MEST, by a grant from the Intramural Research Program (Theragnosis) of KIST, and by a grant (A062254) from the Korea Health 21 R\&D Project.

\section{Conflict of Interest}

The authors have declared that no conflict of interest exists.

\section{References}

1. Lawrence RC, Helmick CG, Arnett FC, Deyo RA, Felson DT, Giannini EH, Heyse SP, Hirsch R, Hochberg MC, Hunder GG, Liang MH, Pillemer SR, Steen VD, Wolfe F. Estimates of the prevalence of arthritis and selected musculoskeletal disorders in the United States. Arthritis Rheum. 1998; 41: 778-99.

2. Andereya S, Streich N, Schmidt-Rohlfing B, Mumme T, Müller-Rath R, Schneider U. Comparison of modern marker proteins in serum and synovial fluid in patients with advanced osteoarthrosis and rheumatoid arthritis. Rheumatol Int. 2006; 26: $432-8$

3. Kikuchi T, Sakuta T, Yamaguchi T. Intra-articular injection of collagenase induces experimental osteoarthritis in mature rabbits. Osteoarthritis Cartilage. 1998; 6: 177-86.

4. Lee S, Park K, Lee SY, Ryu JH, Park JW, Ahn HJ, Kwon IC, Youn IC, Kim K, Choi K. Dark quenched matrix metalloproteinase fluorogenic probe for imaging osteoarthritis development in vivo. Bioconjug Chem. 2008; 19: 1743-7.

5. Hegemann N, Kohn B, Brunnberg L, Schmidt MF. Biomarkers of joint tissue metabolism in canine osteoarthritic and arthritic joint disorders. Osteoarthritis Cartilage. 2002; 10: 714-21.

6. Lai WF, Chang CH, Tang Y, Bronson R, Tung CH. Early diagnosis of osteoarthritis using cathepsin B sensitive near-infrared fluorescent probes. Osteoarthritis Cartilage. 2004; 12: 239-44.

7. Wilder FV, Hall BJ, Barrett JP Jr, Lemrow NB. History of acute knee injury and osteoarthritis of the knee: a prospective epidemiological assessment. The Clearwater Osteoarthritis Study. Osteoarthritis Cartilage. 2002; 10: 611-6.

8. Pavelka K, Forejtová S, Olejárová M, Gatterová J, Senolt L, Spacek P, Braun M, Hulejová M, Stovícková J, Pavelková A. Hyaluronic acid levels may have predictive value for the progression of knee osteoarthritis. Osteoarthritis Cartilage. 2004; 12: $277-83$.

9. Benedetti S, Canino C, Tonti G, Medda V, Calcaterra P, Nappi G, Salaffi F, Canestrari F. Biomarkers of oxidation, inflammation and cartilage degradation in osteoarthritis patients undergoing sulfur-based spa therapies. Clin Biochem. 2010; 43: 973-8.

10. Ahmad R, El Mabrouk M, Sylvester J, Zafarullah M. Human osteoarthritic chondrocytes are impaired in matrix metalloproteinase-13 inhibition by IFN-g due to reduced IFN-g receptor levels. Osteoarthritis Cartilage. 2009; 17: 1049-55.

11. Beekman B, Drijfhout JW, Bloemhoff W, Ronday HK, Tak PP, te Koppele JM. Convenient fluorometric assay for matrix metalloproteinase activity and its application in biological media. FEBS Lett. 1996; 390: 221-5.

12. Smith GN Jr. The role of collagenolytic matrix metalloproteinases in the loss of articular cartilage in osteoarthritis. Front Biosci. 2006; 11: 3081-95.

13. Gupta K, Shukla M, Cowland JB, Malemud CJ, Haqqi TM. Neutrophil gelatinase-associated lipocalin is expressed in osteoarthritis and forms a complex with matrix metalloproteinase 9. Arthritis Rheum. 2007; 56: 3326-35.

14. Makowski GS, Ramsby ML. Zymographic analysis of latent and activated forms of matrix metalloproteinase-2 and -9 in synovial fluid: correlation to polymorphonuclear leukocyte infiltration and in response to infection. Clin Chim Acta. 2003; 329: 77-81.

15. Cawston TE, Weaver L, Coughlan RJ, Kyle MV, Hazleman BL. Synovial fluids from infected joints contain active metalloproteinases and no inhibitory activity. Br J Rheumatol. 1989; 28: 386-92.

16. Ryu JH, Lee A, Lee S, Ahn CH, Park JW, Leary JF, Park S, Kim K, Kwon IC, Youn IC, Choi K. "One-Step" Detection of Matrix Metalloproteinase Activity Using a Fluorogenic Peptide 
Probe-Immobilized Diagnostic Kit. Bioconjug Chem. 2010; 21: 1378-84.

17. Altman R, Asch E, Bloch D, Bole G, Borenstein D, Brandt K, Christy W, Cooke TD, Greenwald R, Hochberg M, et al. Development of criteria for the classification and reporting of osteoarthritis of the knee. Arthritis Rheum. 1986; 29: 1039-49.

18. Honsawek S, Tanavalee A, Sakdinakiattikoon M, Chayanupatkul M, Yuktanandana P. Correlation of plasma and synovial fluid osteopontin with disease severity in knee osteoarthritis. Clin Biochem. 2009; 42: 808-12.

19. Ye $Y$, Chen $X$. Integrin targeting for tumor optical imaging. Theranostics. 2011;1:102-26.

20. Jeong H, Huh M, Lee SJ, Koo H, Kwon IC, Jeong SY, Kim K. Photosensitizer-conjugated human serum albumin nanoparticles for effective photodynamic therapy. Theranostics. 2011;1:230-9.

21. Makowski GS, Ramsby ML. Calibrating gelatin zymograms with human gelatinase standards. Anal Biochem. 1996; 236: 353-6.

22. Makowski GS, Ramsby ML. Zymographic analysis of latent and activated forms of matrix metalloproteinase-2 and -9 in synovial fluid: correlation to polymorphonuclear leukocyte infiltration and in response to infection. Clin Chim Acta. 2003; 329: 77-81.

23. Larsson S, Lohmander LS, Struglics A. Synovial fluid level of aggrecan ARGS fragments is a more sensitive marker of joint disease than glycosaminoglycan or aggrecan levels: a cross-sectional study. Arthritis Res Ther. 2009; 11: R92.

24. Kraus VB, Stabler TV, Kong SY, Varju G, McDaniel G. Measurement of synovial fluid volume using urea. Osteoarthritis Cartilage. 2007; 15: 1217-20.

25. Terp GE, Cruciani G, Christensen IT, Jørgensen FS. Structural differences of matrix metalloproteinases with potential implications for inhibitor selectivity examined by the GRID/CPCA approach. J Med Chem. 2002; 45: 2675-84. 